\title{
Adaptabilidade e estabilidade de populações de cenoura
}

\section{Giovani Olegário da Silva ${ }^{*}$; Agnaldo Donizete F de Carvalho² ${ }^{2}$ Jairo V Vieira ${ }^{2}$; Roberto Fritsche-Neto ${ }^{3}$}

${ }^{1}$ Embrapa Hortaliças-SNT, C. Postal 317, 89460-000 Canoinhas-SC; olegario@cnph.embrapa.br; *autor correspondente; ${ }^{2}$ Embrapa Hortaliças, C. Postal 218, 70351-970 Brasília-DF; agnaldo@cnph.embrapa.br; jairo@cnph.embrapa.br; ${ }^{3} \mathrm{UFV}$, Av. PH Rolfs s/n, 36570-000 Viçosa-MG; rfritscheneto@gmail.com

\section{RESUMO}

No processo de desenvolvimento de novas cultivares de cenoura, é imprescindível o conhecimento do comportamento das populações em fase final de melhoramento, frente aos possíveis ambientes para os quais elas possam vir a ser indicadas. Isso pode ser verificado por meio de análises de adaptabilidade e estabilidade. O objetivo desse estudo foi quantificar a adaptabilidade e estabilidade de populações de cenoura do grupo Brasilia. O trabalho foi conduzido nos anos agrícolas de 2007/2008, 2008/2009 e 2009/2010 em cinco locais: três com cultivo convencional em São Gotardo (MG); Irecê (BA) e Gama (DF); e dois com cultivo orgânico no Programa de Assentamento Dirigido do Distrito Federal (PAD-DF) e em Gama (DF). O delineamento experimental utilizado foi de blocos ao acaso com três repetições e parcelas de $1 \mathrm{~m}^{2}$. Foram avaliadas quatro populações de cenoura do grupo Brasília: 0912532, 0912520, BRS Planalto e Brasília. Aos 100 dias após a semeadura, foi determinada em cada parcela, em gramas, a massa fresca das raízes com padrão comercial. Foi utilizada a metodologia REML/BLUP para a avaliação da adaptabilidade e estabilidade. Pôde-se verificar que as populações com melhor adaptabilidade e estabilidade foram BRS Planalto e 0912520. BRS Planalto destacou-se principalmente na safra de 2009/2010; enquanto a população 0912520 destacou-se em 2007/2008 e 2008/2009; Brasília teve o pior desempenho na maioria dos locais, porém apresentou bom desempenho ou adaptabilidade específica para Irecê(BA).

Palavras-chave: Daucus carota, interação população x ambiente, REML/BLUP.

\begin{abstract}
Adaptability and stability of carrot populations

In the process of developing new cultivars, it is imperative to know the performance of carrot populations in final breeding phase for the possible environments to which they may be indicated; this can be verified testing the adaptability and stability. In this study the adaptability and stability of carrot genotypes from the Brasilia group were verified. The experiment was carried out during the 2007/2008, 2008/2009 and 2009/2010 crop years at five different places, three being in conventional growing at Sao Gotardo (Minas Gerais state), Irece (Bahia state) and Gama (Federal District), and two in organic growing at Managed Settlement Program of the Federal District (PAD-DF) and Gama. A randomized block design with three replications with $1 \mathrm{~m}^{2}$ plots was used. Four carrot genotypes from the Brasilia group: 0912532, 0912520, BRS Planalto and Brasilia were evaluated. 100 days following sowing, fresh mass of commercial root value was determined in grams, in each plot. Regarding adaptability and stability assessment, the REML/BLUP methodology was applied. BRS Planalto and 0912520 were the genotypes with better adaptability and stability; BRS Planalto was preeminent mainly in 2009-2010 crop years; and 0912520 was preeminent in 2007/2008 and $2008 / 2009$. Brasilia had the worst performance in most places, but good performance and specific adaptability to Irece.
\end{abstract}

Keywords: Daucus carota, population x environment interaction, REML/BLUP.

(Recebido para publicação em 3 de dezembro de 2010; aceito em 8 de fevereiro de 2012) (Received on December 3, 2010; accepted on February 8, 2012)

$\mathrm{A}$ cenoura é uma hortaliça da família Apiaceae, do grupo das raízes tuberosas, cultivada em larga escala nas regiões Sudeste, Nordeste e Sul do Brasil. Aárea plantada no Brasil é cerca de 28 mil hectares com produção de 750 mil toneladas de raízes. Embora produza melhor em áreas de clima ameno, nos últimos anos, face ao desenvolvimento de cultivares tolerantes ao calor e com resistência às principais doenças de folhagem, o plantio de cenoura vem-se expandindo também nos estados da Bahia e Goiás. Esta olerícola apresenta alto conteúdo de vitamina A (12000 UI/100 g), textura macia e paladar agradável. Além do consumo in natura, é utilizada como matéria prima para indústrias processadoras de alimentos (Embrapa Hortaliças, 2011).

No processo de desenvolvimento de novas cultivares de cenoura, é imprescindível o conhecimento do comportamento das populações em fase final de melhoramento frente aos possíveis ambientes para os quais elas possam vir a ser indicadas; isso pode ser verificado através de análises de adaptabilidade e estabilidade.

O desenvolvimento das plantas é resultado do efeito do ambiente (A), população $(G)$ e da interação entre ambos (GxA), que resulta em significativas diferenças no desempenho das cultivares quando são cultivadas em diferentes condições ambientais (Cruz
\& Regazzi, 2001). Nesse sentido, algumas populações de plantas possuem adaptação ampla enquanto outras são restritas a determinadas condições ambientais para o cultivo. A presença da GxA interfere significativamente nos programas de melhoramento, pois numa situação ideal uma cultivar deveria ser adaptada a um ambiente amplo de cultivo, porém o fator interação faz com que na maioria das vezes o lançamento de cultivares seja realizado para ambientes específicos onde estas possuem maior adaptação (Campbell \& Jones, 2005). Apesar de ser mais cômodo para o lançamento de cultivares, a ausência da GxA é um grande problema em termos de ganhos com a seleção. Sabe-se que 
esta é herdável e pode ser capitalizada, maximizando os ganhos genéticos para ambientes específicos.

Espera-se sempre que uma cultivar tenha boa adaptabilidade e estabilidade. O termo adaptabilidade refere-se à capacidade das populações aproveitarem vantajosamente o estímulo do ambiente, enquanto estabilidade refere-se à capacidade das populações desempenharem um comportamento previsível em função do estímulo do ambiente (Cruz \& Regazzi, 2001). Diversos métodos têm sido propostos para avaliar a adaptabilidade e estabilidade produtiva sobre GxA significativa, sendo exemplos as metodologias de Wricke, Eberhart \& Russel e Lin \& Binss (Cruz \& Regazzi, 2001). Entretanto, tem-se observado em diversas espécies que o comportamento das populações não é satisfatoriamente explicado por esses métodos tradicionais (Duarte \& Zimmerman, 1994; Namorato et al., 2009; Osmanzai \& Sharma, 2008; Zobel et al., 1988).

Buscando contornar este problema uma metodologia recente e que tem apresentado bons resultados é a metodologia baseada nas equações de modelos mistos (REML/BLUP - Máxima Verossimilhança Residual ou Restrita/Melhor Predição Linear Não Viciada).

Esta análise baseia-se nas seguintes estimativas: quanto menor for o desvio-padrão do comportamento genotípico através dos locais, maior será a média harmônica de seus valores genotípicos através dos locais. Assim, a seleção pelos maiores valores da média harmônica dos valores genotípicos (MHVG) implica simultaneamente seleção para produtividade e estabilidade. Em termos de adaptabilidade, refere-se ao desempenho relativo dos valores genotípicos (PRVG) através dos ambientes. Neste caso, os valores genotípicos preditos (ou os dados originais) são expressos como proporção da média geral de cada local e, posteriormente, obtém-se o valor médio dessa proporção através dos locais. A seleção simultânea por produtividade, estabilidade e adaptabilidade, no contexto dos modelos mistos, pode ser realizada pelo método da média harmônica do desempenho relativo dos valores genéticos (MHPRVG) preditos. Esse método permite selecionar simulta- neamente pelos três atributos mencionados e apresenta as seguintes vantagens: (a) considera os efeitos genotípicos como aleatórios e, portanto fornece estabilidade e adaptabilidade genotípica e não fenotípica; (b) permite lidar com desbalanceamento; (c) permite lidar com delineamentos não ortogonais; (d) permite lidar com heterogeneidade de variâncias; (e) permite considerar erros correlacionados dentro de locais; (f) fornece valores genéticos já descontados (penalizados) da instabilidade; $(\mathrm{g})$ pode ser aplicado com qualquer número de ambientes; (h) permite considerar a estabilidade e adaptabilidade na seleção de indivíduos dentro de progênie; (i) não depende da estimação de outros parâmetros tais quais coeficientes de regressão; (j) gera resultados na própria grandeza ou escala do caráter avaliado; (k) permite computar o ganho genético com a seleção pelos três atributos simultaneamente (Resende, 2002a).

A utilização da metodologia REML/ BLUP para este tipo de estimativa em cenoura é inédita na literatura.

O objetivo desse estudo foi quantificar a adaptabilidade e estabilidade de populações de cenoura de verão grupo Brasilia.

\section{MATERIAL E MÉTODOS}

O presente estudo foi conduzido nos anos agrícolas de 2007/08, 2008/09 e 2009/10 em cinco locais, três com cultivo convencional São Gotardo (MG); Irecê (BA) e Gama (DF); e dois com cultivo orgânico Programa de Assentamento Dirigido (PAD-DF) e Gama (DF).

Utilizou-se o delineamento experimental de blocos ao acaso com três repetições. Foram avaliadas quatro populações de cenoura do grupo Brasilia: duas populações 0912532 e 0912520 e duas cultivares BRS Planalto e Brasilia. As parcelas constituíram-se de $1 \mathrm{~m}^{2}$, com espaçamento de $20 \mathrm{~cm}$ entre linhas e $5 \mathrm{~cm}$ entre plantas. Aos 100 dias após a semeadura foi determinada em cada parcela, em gramas, a massa fresca das raízes comercializáveis de acordo com o padrão proposto pela Ceagesp (1999), não sendo considerado como raízes comerciais as que apresentaram diâmetro do terço médio superior inferior a $2 \mathrm{~cm}$.
A avaliação da adaptabilidade e estabilidade foi realizada por meio da metodologia REML/BLUP(Henderson, 1975), considerando o seguinte modelo estatístico:

$$
Y=X_{r}+Z_{g}+W_{i}+e
$$

Em que: Y é o vetor de dados, $r$ é o vetor dos efeitos de repetição (assumidos como fixos) somados à média geral, e contempla todas as repetições de todos os locais, $g$ é o vetor dos efeitos genotípicos (assumidos como aleatórios), $i$ é o vetor dos efeitos da interação populações x locais (aleatórios), sendo $e$ o vetor de erros (aleatórios). As letras maiúsculas representam as matrizes de incidência para os referidos efeitos.

Para avaliação genética pelos maiores valores da média harmônica dos valores genotípicos foi utilizado o método MHPRVG, conforme descrito por Resende (2002a).

Para a realização das análises foi utilizado o aplicativo computacional Selegen (Resende, 2002b).

\section{RESULTADOS E DISCUSSÃO}

A análise de deviance em relação à massa fresca de raízes comerciais (Tabela 1) revelou os efeitos de populações como não significativos; o que é normal em análises conjuntas considerando ambientes contrastantes. O coeficiente de variação foi de $13,61 \%$ conferindo boa precisão ao experimento, já que o caráter massa fresca de raiz comerciais é altamente influenciado pelo ambiente (Vieira \& Silva, 2008; Vieira et al., 2009), sendo este valor semelhante aos obtidos por Oliveira et al. (2005) e abaixo dos obtidos por Oliveira et al. (2008).

A interação entre populações e ambientes foi significativa, indicando que as populações apresentaram respostas diferenciadas quando submetidas aos diferentes ambientes (locais e safras (anos)) de produção, revelando que ocorreu alteração no posicionamento das populações ou mudanças na magnitude das diferenças entre as populações e os ambientes estudados.

$\mathrm{Na}$ Tabela 2 é possível visualizar as populações com os respectivos valores genotípicos para cada local de cultivo e 
Tabela 1. Valores da estatística do teste razão de verossimilhança (LRT) da análise de deviance conjunta, média geral e coeficiente de variação experimental $(\mathrm{CV}(\%))$ para massa fresca de raiz comercial de populações de cenoura avaliadas em cinco locais, nas safras agrícolas 2007/2008, 2008/2009 e 2009/2010 (values of the statistical likelihood ratio test (LRT) of the joint deviance analysis, general average and coefficient of experimental variation $(\mathrm{CV}(\%))$ for fresh mass of commercial root of carrot populations tested at five locations, in the 2007/2008, 2008/2009 and 2009/2010 crops year). Brasília, Embrapa Hortaliças, 2010.

\begin{tabular}{lc}
\hline Efeitos $^{\mathbf{1}}$ & Massa fresca de raiz comercial \\
\hline Populações & $1,00^{\text {ns }}$ \\
Populações x ambientes & $4,34 * *$ \\
\hline CV $(\%)$ & 13,61 \\
Média geral & 5620 \\
\hline
\end{tabular}

${ }^{1}$ Valores de LRT; significativo a ${ }^{* *} \mathrm{p}=0,05$ e ${ }^{\text {ns }}$ não significativo pelo teste $\chi 2$ com 1 grau de liberdade. ( ${ }^{1}$ LRT values, ${ }^{* *}$ significant at $\mathrm{p}=0.05$ and not significant by $\chi 2$ test with one degree of freedom).

Tabela 2. Valores genotípicos pela metodologia REML/BLUP para massa fresca de raiz comercial de populações de cenoura avaliadas em cinco locais, nas safras agrícolas 2007/2008, 2008/2009 e 2009/2010 (genotypic values by the methodology REML/BLUP for fresh mass of commercial root of carrot populations tested at five locations, in the 2007/2008, 2008/2009 and 2009/2010 crop years). Brasília, Embrapa Hortaliças, 2010.

\begin{tabular}{lrrrr}
\hline & \multicolumn{4}{c}{ População de cenoura } \\
\cline { 2 - 5 } Local & $\mathbf{0 9 1 2 5 3 2}$ & $\mathbf{0 9 1 2 5 2 0}$ & BRS Planalto & Brasilia \\
\cline { 2 - 5 } & $-24,81$ & 255,90 & 101,48 & $-332,56$ \\
\hline São Gotardo-MG & 211,31 & 498,66 & 108,74 & $-818,71$ \\
PAD-DF & $-138,14$ & $-19,77$ & $-38,81$ & 196,72 \\
Gama-DF orgânico & 138,36 & 19,16 & 161,63 & $-319,15$ \\
Gama-DF & $-237,25$ & $-135,90$ & 174,59 & 198,56 \\
Irecê-BA & $-10,11$ & 123,61 & 101,52 & $-215,03$ \\
Média de 2007/2008 & \multicolumn{4}{c}{ Safra 2008/2009 } \\
\hline & $-78,05$ & 246,63 & $-53,40$ & $-115,18$ \\
\hline São Gotardo-MG & 4,23 & 73,09 & 210,03 & $-287,34$ \\
PAD-DF & 393,85 & 96,25 & $-206,55$ & $-283,54$ \\
Gama-DF orgânico & $-70,45$ & 346,89 & 156,10 & $-432,54$ \\
Gama-DF & $-27,58$ & $-155,08$ & 24,73 & 157,93 \\
Irecê-BA & 44,40 & 121,56 & 26,18 & $-192,14$ \\
Média de 2008/2009 & \multicolumn{4}{c}{ Safra 2009-2010 } \\
\hline & $-147,20$ & $-25,79$ \\
\hline São Gotardo-MG & $-44,52$ & 25,72 & 211,07 & $-192,27$ \\
PAD-DF & 18,06 & $-184,12$ & 204,50 & $-38,43$ \\
Gama-DF orgânico & 89,27 & 47,50 & 220,40 & $-357,18$ \\
Gama-DF & $-312,79$ & $-17,14$ & 118,42 & 211,52 \\
Irecê-BA & $-79,44$ & $-30,77$ & 152,64 & $-42,44$ \\
Média de 2009/2010 & $-8,14$ & 38,64 & 50,53 & $-81,03$ \\
Média de todos os ambientes & \multicolumn{4}{c}{} \\
\hline
\end{tabular}

em conjunto para todos os locais para cada safra agrícola. Pode-se verificar que a cultivar Brasilia teve o menor valor genotípico médio, ou seja, teve o pior desempenho na média dos locais e safras. Considerando os valores genotípicos, as melhores cultivares ou populações no conjunto de safras agrícolas e locais foram BRS Planalto e 0912520. A cultivar BRS Planalto foi a mais adaptada ao conjunto de locais na safra de 2009-2010, enquanto a população 0912520 se destacou nos demais anos.

A população 0912532 apresentou variações nos diferentes anos, mas no geral mostrou maiores valores genotípicos no PAD-DF e Gama sob cultivo orgânico e no Gama, sob cultivo convencional. A população 0912520 apresentou pouca variação nas duas primeiras safras, com bom desempenho no PAD, Gama e em São Gotardo. BRS Planalto apresentou bom desempenho em PAD-DF, Gama e Irecê; e Brasilia apresentou os maiores valores genotípicos especificamente em Irecê, em todos os anos (Tabela 2), indicando ter adaptabilidade especificamente para este local, região esta que atualmente é a maior produtora desta cultivar de cenoura no Brasil.

$\mathrm{Na}$ Tabela 3 estão apresentados os resultados esperados em gramas de massa fresca de raízes comerciais por metro quadrado, penalizando ou capitalizando as populações de acordo com seu desempenho em relação à estabilidade (MHVG), adaptabilidade (PRVG), e estabilidade e adaptabilidade conjuntamente (MHPRVG) para o conjunto de locais.

Tanto em relação à estabilidade quanto para adaptabilidade, verifica-se que a cultivar BRS Planalto e a população 0912520 foram superiores às demais, concordando com as estimativas de valores genotípicos apresentados anteriormente. A população com menor estabilidade e adaptabilidade foi Brasilia.

Estudando a adaptabilidade e estabilidade da cultivar Brasilia em regiões dos estados de São Paulo, Minas Gerais e Paraná, Oliveira et al. (2005) verificaram que esta cultivar respondeu medianamente a ambientes favoráveis e se destacou pela tolerância à queima-das-folhas. O mesmo foi verificado por Oliveira et al. (2008), avaliando esta mesma cultivar em várias épocas e espaçamentos de semeadura em São José do Rio Pardo (SP).

Desta forma, pôde-se verificar que as populações com melhores desempenhos nos ambientes estudados e consequentemente maior adaptabilidade e estabilidade foram BRS Planalto e 0912520; a cultivar BRS Planalto foi a mais adaptada ao conjunto de locais na safra de 2009/2010, enquanto a população 0912520 se destacou nos demais anos 
Tabela 3. Estabilidade de valores genotípicos (MHVG), adaptabilidade de valores genotípicos (PRVG), estabilidade e adaptabilidade de valores genotípicos (MHPRVG) para o caráter massa de raízes comerciais de populações de cenoura avaliados em cinco locais, nas safras agrícolas 2007/2008, 2008/2009 e 2009/2010 (stability of genotypic values (MHVG), adaptability of genotypic values (PRVG), stability and adaptability of genotypic values (MHPRVG) for the character mass of commercial roots of carrot genotypes tested at five locations, in the 2007/2008, 2008/2009 and 2009/2010 crop years). Brasília, Embrapa Hortaliças, 2010.

\begin{tabular}{lccc}
\hline \multirow{2}{*}{ População de cenoura } & \multicolumn{3}{c}{ Valores genotípicos } \\
\cline { 2 - 4 } & MHVG & PRVG & MHPRVG \\
\hline 0912532 & $4.304,29$ & $5.622,70$ & $5.616,14$ \\
0912520 & $4.368,16$ & $5.717,92$ & $5.702,27$ \\
BRS Planalto & $4.474,74$ & $5.783,25$ & $5.776,81$ \\
Brasilia & $3.980,01$ & $5.358,30$ & $5.320,92$ \\
\hline
\end{tabular}

e, Brasilia teve o pior desempenho na média dos locais, porém apresentou bom desempenho ou adaptabilidade específica para Irecê-BA. Estas informações são muito importantes, pois possibilitam a recomendação de cultivo e verificação do desempenho das populações frente às regiões produtoras. Além disso, indicam que a seleção de populações deve ser realizada para ambientes específicos buscando com isto capitalizar a interação.

\section{REFERÊNCIAS}

CAMPBELL BT; JONES MA. 2005. Assessment of genotype $\mathrm{x}$ environment interactions for yield and fiber quality in cotton performance trials. Euphytica 144: 69-78.

CEAGESP. 1999. Classificação de cenoura: programa de adesão voluntária (Folder), São Paulo: Programa Horti \& Fruti. 8p.

CRUZ CD; REGAZZI AJ. 2001. Modelos biométricos aplicados ao melhoramento genético. Viçosa: UFV. 390p.

DUARTE JB; ZIMMERMAN MJO. 1994. Adaptabilidade e estabilidade de rendimento de populações de feijão comum. Pesquisa Agropecuária Brasileira 29: 25-32.

EMBRAPA HORTALIÇAS. 2011, 13 de abril. Hortaliças em números. Disponível em: http:// www.cnph.embrapa.br/paginas/hortalicas em numeros.htm.

HENDERSON CR. 1975. Best linear estimation and prediction under a selection model. Biometrics 31: 423-447.
NAMORATO H; MIRANDA GV; SOUZA LV; OLIVEIRA LR; DELIMA RO; MANTOVANI EE. 2009. Comparing biplot multivariate analyses with Eberhart and Russel' method for genotype $\mathrm{x}$ environment interaction. Crop Breeding and Applied Biotechnology 9: 299-307.

OLIVEIRA CD; BRAZ LT; BANZATTO DA. 2005. Adaptabilidade e estabilidade genotípica de populações de cenoura. Horticultura Brasileira 23: 743-748.

OLIVEIRA CD; BRAZ LT; BANZATTO DA. 2008. Adaptabilidade e estabilidade fenotípica de cultivares de cenoura. Horticultura Brasileira 26: 88-92.

OSMANZAI M; SHARMA RC. 2008. High yielding stable wheat genotypes for the diverse environments in Afghanistan. International Journal of Agricultural Research 3: 340-348.

RESENDE MDV. 2002a. Genética biométrica e estatística no melhoramento de plantas perenes. Brasilia: Embrapa Informação Tecnológica. 975p.

RESENDE MDV. 2002b. Software SelegenREML/BLUP. Curitiba: Embrapa Florestas, $67 \mathrm{p}$.

VIEIRA JV; SILVA GO. 2008. Tamanho mínimo de parcela para avaliação de caracteres de raiz em cenoura. Bragantia 67: 817-825.

VIEIRA JV; SILVA GO; BOITEUX LS; SIMON P. 2009. Divergência genética entre acessos de cenoura pertencentes a grupos varietais distintos utilizando caracteres morfológicos. Horticultura Brasileira 27: 468-472.

ZOBEL RW; WRIGHT MJ; GAUCH HG. 1988. Statistical analysis of a yield trial. Agronomy Journal 80: 388-393. 